\title{
Characterizing and designing photopolymer materials
}

\section{John T. Sheridan}

Understanding how photopolymers react to light exposure may fuel developments in holographic data storage.

Photopolymers are organic materials that polymerize when illuminated with a suitable wavelength of light. They have many potential applications, such as optically written waveguides for $3 \mathrm{D}$ photonic circuits. Perhaps the most exciting applications are in the area of data storage.

Holographic data storage has been identified as a significant goal, and photopolymers are the medium of choice for the digital versatile disc (DVD)-sized devices with terabyte-plus data densities promised by these 3D optical storage techniques. High-density magnetic tapes can currently hold 500GB (approximately 200 million pages of text). Magnetic tape libraries, sold to governments and large companies, are made by connecting cabinets that hold $\sim 5,000$ tapes. Companies like StorageTech sell over 22,000 of these cabinets annually, so the need for high-volume storage exists. Several important technical milestones have recently been achieved in this area, such as InPhase Technologies launching their holographic disc-drive in 2008. The commercial possibilities may explain why several companies, including Bayer, DuPont, General Electric, and Sony have entered this research area.

Since $2000,{ }^{1}$ our group has been developing detailed electromagnetic and material models to describe the operation of photopolymers. $^{2-5}$ The aim has been to develop simulations ${ }^{4}$ that characterize the physical and chemical effects taking place within the material during and after exposure. These efforts could allow scientists to optimize materials and simulate their applications.

Figure 1 shows the material processes involved. Exposing light produces a sinusoidal interference pattern. The grating causes diffraction of the probing beam in the non-latent material. Polymerization (initiated in the bright regions of the material layer) removes the monomer, producing a concentration gradient. The monomer diffuses from the dark to the bright regions. Non-local growth of the polymer chains (away from the point of

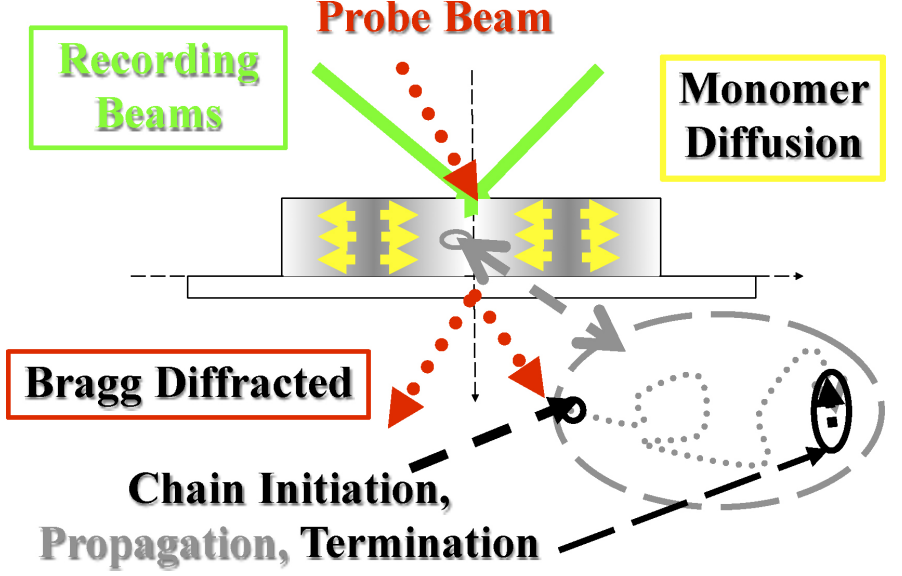

Figure 1. Material processes occurring in photopolymers during and after exposure

initiation) leads to a smeared recording of the exposing pattern and loss of fidelity and resolution.

Understanding initiation involves detailed knowledge of the absorptive, recovery, and bleaching properties of the dye used. ${ }^{5}$ In $2008^{3}$ we applied our model to chemically improve the performance of a photopolymer by controlling the chain length (reducing the average molecular weight), and, thus, localizing the effects of the polymer chains formed.

Most recently, we used our model to analyze photopolymers sensitized with different dyes and chemical compositions. For a material composed of an acrylamide monomer in a polyvinylalcohol-based matrix, we compared experimental measurements and theoretical predictions of grating refractive index modulations $\left(n_{1}\right)$ for three exposures (see Figure 2). Initial inhibition (deadband), exposure growth, and post-exposure effects (dark reactions) are all visible. A reduction in grating modulation occurs post-exposure because the monomer has a lower refractive index than the layer. As the monomer diffuses from the dark to the bright regions, it lowers the grating modulation strength. By contrast, for a short exposure in an epoxy resin

Continued on next page 


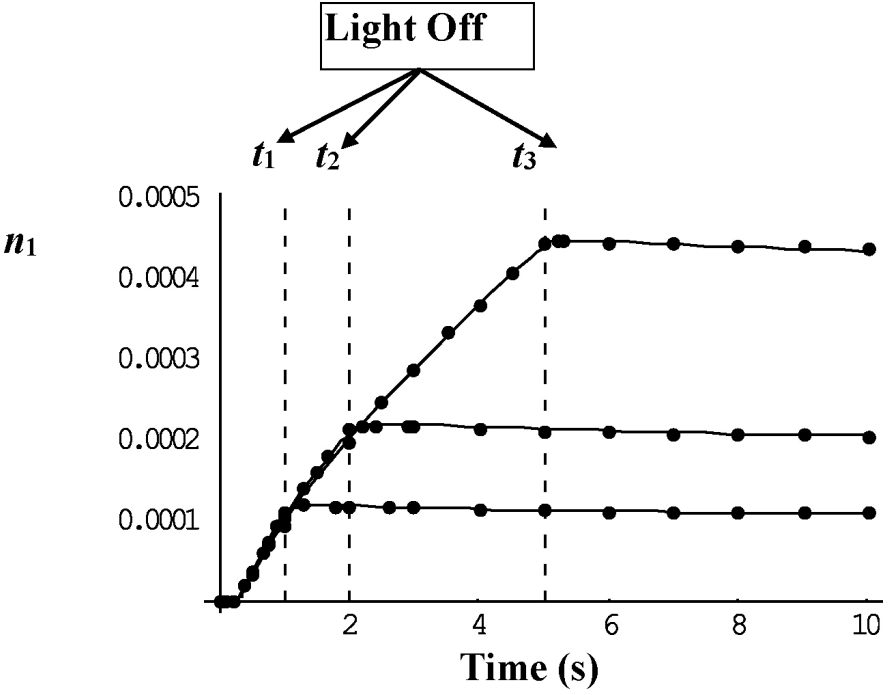

Figure 2. Experimentally measured grating refractive index modulation (dots) and theoretical fits (lines) for three exposures $\left(t_{1}, t_{2}\right.$, and $t_{3}$ ) in a material consisting of an acrylamide monomer in a polyvinylalcohol-based matrix.

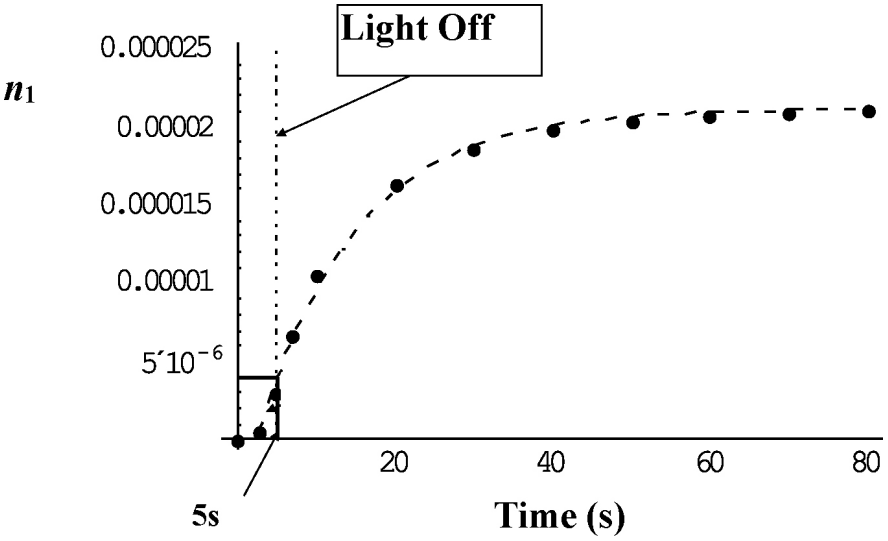

Figure 3. Experimental data (dots) and theoretical fit (long dashed line) for a short exposure in the epoxy resin photopolymer material. In this case the monomer has a higher refractive index than the layer, so diffusion after exposure increases the grating strength.

photopolymer material (see Figure 3), the monomer has a higher refractive index than the layer and therefore post-exposure diffusion increases the grating strength.

Much of this work ${ }^{1-5}$ was independently verified in the academic literature. In 2007, Sony researchers used our non-local photopolymerization-driven diffusion model in the development of their materials and systems. In 2008, we began a collaboration with Bayer Material Science (fully funded by the company) to apply our analysis to develop their new range of photopolymer material products. While several of the remaining problems raised ${ }^{4}$ have been addressed, ${ }^{6,7}$ others, including full 3D- and multi-component modeling, are currently being more thoroughly examined.

This work has been supported by Enterprise Ireland; Science Foundation Ireland; The Irish Research Council for Science, Engineering and Technology; The Irish National Training and Employment Authority (FAS); and The Irish Canadian Fund.

\section{Author Information}

\section{John T. Sheridan}

School of Electrical, Electronic and Mechanical Engineering University College Dublin

and

National University of Ireland

Dublin, Ireland

John T. Sheridan is a professor of optical engineering. He has authored over 300 technical publications and is a member of the editorial boards of several international journals (including SPIE Review) and of several SPIE conference committees. His current research interests include optical signal processing, holography, and photopolymer materials.

\section{References}

1. J. T. Sheridan and J. R. Lawrence, Non-local response diffusion model of holographic recording in photopolymer, J. Opt. Soc. Amer. A 17 (6), pp. 1108-1114, 2000.

2. J. T. Sheridan, M. R. Gleeson, C. E. Close, and J. V. Kelly, Optical response of photopolymer materials for holographic data storage applications, J. Nanosci. Nanotech. 7 (1), pp. 232-242, 2007.

3. M. R. Gleeson, D. Sabol, S. Liu, C. E. Close, J. V. Kelly, and J. T. Sheridan, Improvement of the spatial frequency response of photopolymer materials by modifying polymer chain length, J. Opt. Soc. Amer. B 25 (3), pp. 396-406, 2008.

4. M. R. Gleeson and J. T. Sheridan, A review of the modeling of free-radical photopolymerization in the formation of holographic gratings, J. Opt. A: Pure Appl. Opt. 11, p. 12, 2009.

5. S. Liu, M. R. Gleeson, and J. T. Sheridan, Analysis of the photo-absorptive behaviour of two different photosensitizers in a photopolymer material, J. Opt. Soc. Amer. B. 26 (3), pp. 528-536, 2009

6. M. R. Gleeson and J. T. Sheridan, Nonlocal photopolymerization kinetics including multiple termination mechanisms and dark reactions. Part I. Modeling, J. Opt. Soc. Am. B 26 (9), pp. 1736-1745, 2009.

7. M. R. Gleeson, S. Liu, R. R. McLeod, and J. T. Sheridan, Nonlocal photopolymerization kinetics including multiple termination mechanisms and dark reactions. Part II. Experimental validation, J. Opt. Soc. Am. B 26 (9), pp. 1746-1754, 2009. 